MULTIPLE SCLEROSIS

\title{
Real-world long-term benefits of disease-modifying MS therapy
}

Disease-modifying therapies (DMTs) for relapsing-remitting multiple sclerosis (MS) have long-term benefits in the real world, a new large registry cohort study has shown. The analysis also revealed that a high annualized relapse rate, particularly on treatment, is associated with a poor prognosis, and that pregnancy protects against disability progression.

Much of the evidence that DMTs are effective in MS has come from clinical trials, which do not necessarily reflect the long-term benefits of treatment in the real world. Large data registries offer the opportunity to study real-world outcomes in large cohorts. Vilija Jokubaitis and an international team took this approach to gain insight into the real-world benefits of first-line DMTs in MS.

"There has been some data published that suggest these first-line drugs do not protect against disability worsening in the long-term," says Jokubaitis. "We decided to use data from our prospective, longitudinal outcomes registry MSBase to determine whether first-line DMT has a long-term benefit in MS."

Jokubaitis and colleagues examined the records of 2,466 patients with MS who had been followed up for at least 10 years after the initiation of injectable DMT. The patients were treated with IFN- $\beta$ or glatiramer acetate, and were treated for a mean of $83 \%$ of their follow-up period. The research team assessed the effects of DMTs on disability by comparing Expanded Disability Status Scale (EDSS) scores at baseline (the initiation of DMTs) and at 10 years.

The median increase in EDSS scores was 1 point, but use of DMTs was associated with a smaller increase in EDSS score. This effect was at a level that would prevent an increase in EDSS score of 1 point for every 11.6 years of treatment.

The researchers also looked at factors that could predict the long-term change in EDSS score, and found that annualized relapse rate was the most robust predictor. "More significantly, we found that not all relapses are equal," Jokubaitis adds. "Relapses that occurred while on therapy carried a much greater disability burden than those that occurred off therapy." The analysis also revealed that one or more pregnancies during the follow-up period protected women against the accrual of disability.

"As more follow-up data become available, we'd like to assess the longterm impact of newer-generation therapies for which there is insufficient long-term data for assessment," says Jokubaitis. "We would also like to pursue the pregnancy finding further: we are confident that women with relapse MS who take injectable therapies should not be discouraged from pregnancy, but we need to determine whether this also extends to more severely affected women."

\section{Ian Fyfe}

ORIGINAL ARTICLE Jokubaitis, V. G. et al. Predictors of long-term disability accrual in relapse-onset multiple sclerosis. Ann. Neurol. http://dx.doi.org/10.1002/ana.24682 (2016) 